\title{
Effective technical ways to improve the vibro-centrifugal separator electric drive for grain cleaning
}

\author{
Andrey Linenko, ${ }^{1}$ Bulat Khalilov, ${ }^{2}$ Timur Kamalov, ${ }^{2}$ Marat Tuktarov, ${ }^{1}$ Damir Syrtlanov ${ }^{1}$ \\ ${ }^{1}$ Department of Electric Machines and Equipment; ${ }^{2}$ Department of Electricity Supply and Automation of Technological \\ Processes, Federal State Budgetary Educational Establishment of Higher Education 'Bashkir State Agrarian \\ University', Ufa, Russia
}

\begin{abstract}
Grain production economic efficiency mostly depends on the use of energy-efficient, resource-saving technological equipment that can ensure a high-quality technological process. In this regard, this paper considers ways to improve the vibrating drives of vibration-centrifugal grain separators which have a complex design and involve high operating expenses (the life of vibrator bushing does not exceed $180 \pm 20$ hours). A linear induction motor was used in this study as a vibrating drive, which directly forwarded working body movement without using any movement converters. This type of motor together with elastic elements helps to implement energy-efficient electric vibrating motion with adjustable vibration parameters. The mathematical model of the vibro-centrifugal grain separator with a linear motor was developed. The model was implemented in the environment of MatLab (Simulink) object-visual modelling, which showed the correlations of the working body vibration parameters with the drive kinematic parameters. The suitability of the model was proved experimentally. The difference between the experimental data and the data obtained by mathematical modelling does not exceed $6 \%$. The comparative assessment results showed that the maintenance intervals increased by $37.8 \%$ in the project version compared to the basic version. The proposed technical solution made it possible to obtain the vibrational motion of the centrifuge working body using the linear induction electric motor without the need for
\end{abstract}

Correspondence: Andrey Linenko, Department of Electric Machines and Equipment, Federal State Budgetary Educational Establishment of Higher Education 'Bashkir State Agrarian University', 50-letia Octyabrya Str., 34, 450001, Ufa, Russia.

E-mail: a.linenko542@rambler.ru

Key words: Separator; cleaning; linear motor; electric drive; vibrations; grain separator.

Received for publication: 9 November 2020.

Accepted for publication: 19 March 2021.

(C) Copyright: the Author(s), 2021

Licensee PAGEPress, Italy

Journal of Agricultural Engineering 2021; LII:1136

doi:10.4081/jae.2021.1136

This article is distributed under the terms of the Creative Commons Attribution Noncommercial License (by-nc 4.0) which permits any noncommercial use, distribution, and reproduction in any medium, provided the original author(s) and source are credited. a control unit to connect and disconnect it periodically from the mains. This can reduce the starting currents in the inductor windings and increase the reliability of the installation. Thanks to the use of the linear induction electric motor in the vibrating drive of the vibro-centrifugal separators vibrating drive, it is also possible to save metal.

\section{Introduction}

In a competitive market economy, two key factors for successful production are the reduction of production costs and the improvement of work quality. These factors are also important for farms and are directly related to the development and implementation of new systems, technologies, and machines designed to perform various technological processes. These requirements are of particular importance for the intensification of widely used technological processes, including grain mixture separation which occurs during grain harvesting, storage, and processing. Every year, in all countries across the world millions of tons of grain crops undergo the separation process. Therefore, even a slight improvement in some technological process parameters, such as energy intensity and separation efficiency, can have a significant economic impact (Al Maidi, 2015; Ostrikov et al., 2019).

The annual growing demand for grain production requires the use of high-capacity machinery that combines low operating expenses, production cost, and high productivity (Panasiewicz et al., 2012; Al Maidi, 2015; Khasanov et al., 2019). The industry offers a large number of different types of grain separators. Flat sieve separators are most widely used in grain storage and processing facilities. One of the main difficulties for further improvement and increase of the efficiency of flat sieve separation is the use of gravity forces when separating grain mixtures (Iarullin, 2007).

Centrifugal separation is a more efficient and productive method for grain separation. Centrifugal separators differ in design, shape, and working body location. Drum-type separators with a horizontal rotation axis are the most widely used. The disadvantages of these grain separators are the low quality of the grain mixture separation due to the low sieve use coefficient and the uneven thickness of the grain mixture layer on the sieve. Moreover, the sieves of the lower part of the working body where the separation takes place are not cleaned from trapped particles in these machines (Iarullin, 2007; Liu et al., 2014).

The most promising grain-cleaners are vibro-centrifugal separators (VCS), in which intensive grain mixture separation occurs due to inertial forces of rotational and vibrational working body movements (Tishchenko et al., 2010). Constant contact with the separating surface increases the probability of having particles 
enter through the sieve holes, and the high speed of the separated material ensures high productivity of separators. Compared with the separation of the grain mixture using gravity forces (in flatsieve machines), the propulsion of oscillatory motion to the working body along the rotation axis has made it possible to increase separation efficiency. The productivity of these separators compared to flat-sieve machines with the same sieve surface area is 4 times higher, while their energy consumption is 2 times lower. Due to the vertical sieve arrangement, they occupy less working space, and require 5 times less metal for their structure (Iarullin, 2007).

The existing design of the oscillatory drive of the VCS working body based on the motor rotational motion with a crank mechanism as a motion transformer has several disadvantages, namely, the complex kinematic scheme of the working body movement along the vertical plane and the impossibility of regulating the working body oscillation amplitude without replacing the functional elements of the oscillatory drive. The above-mentioned disadvantages lead to increased wear of the vibrating drive elements (bushings, vibrator bearings, etc.), which have a service life of about $180 \pm 20$ hours. As a result, the VCS efficiency drops (Linenko et al., 2018, 2019).

One way to overcome these disadvantages is to design a vibrating grain separator drive based on a flat linear induction electric motor (LIM), which converts electrical energy into the translational motion of the secondary element without using any motion converters. The LIM consists of an inductor (stator) and a secondary element (rotor). In such engines, the secondary element can also be the working body of the technological machine itself (Ganesh Sampath et al., 2016; Pathan et al., 2017; Vinod, 2017). Therefore, electrical energy is immediately converted into the translational motion of the working body (Alanis et al., 2015; Jiao et al., 2017; Lim et al., 2017). The electric motor efficiency factor in such drives is equal to the efficiency factor of an electric drive. In rotation motor drives, the drive efficiency factor can fall several times compared to the rotation engine efficiency factor (Ahmadinia, 2014; Sannomiya et al., 2017; Linenko et al., 2018, 2019).

The use of elastic mechanical energy storage units (helical springs) in drives with a LIM can significantly increase their efficiency, since, during the working body straight movement, the accumulated kinetic energy is not extinguished by the plugging mode, as it is converted into the potential energy of the elastic element. This energy is then returned to the working body. Depending on the number of elastic elements and their connection with the working body, various kinematic schemes of oscillatory electric drives based on a linear induction motor make it possible to implement an energy-efficient oscillatory motion electric drive (Aipov and Linenko, 2013).

There are known cases in which the LIMs were used with elastic energy storage units for such technological machines as flatsieve separators, shelter machines, inertial conveyors of wet sugar, burr mills, feed bins, subsurface pumps, etc. (Aipov and Linenko, 2013).

A promising direction for the development of an electric oscillatory motion drive is leading to mechatronic systems based on the LIM, in which an electric machine is joined to the working body by an intelligent control system. The use of an expensive LIM control system leads to an increase in the cost of the working body oscillatory movement drive and reduces its reliability. Therefore, it is urgent to develop new effective technical solutions to ensure the rotational-oscillatory movement of the working body without having to use a specific system to control the working body vibrations (Linenko et al., 2018, Ostrikov et al., 2020).

The application of LIMs for VCSs, which were not previously used for rotating mechanisms, will eliminate the above-mentioned disadvantages, since the design of the bearing unit will be significantly simplified, and their working conditions will become easier, and improve the efficiency of the vibration-centric grain separator.

\section{Objectives}

Research objectives were to: i) consider possible ways of improving the design of the VCS vibrating drive based on a linear induction electric motor with adjustable working body vibration parameters; ii) develop a mathematical model of the proposed VCS vibrating drive with a LIM; iii) make an experimental assessment of the drive frequency characteristics and confirm the suitability of the developed mathematical model.

\section{Materials and methods}

A vibration motion of the rotating working body along the vertical plane achieved using the LIM will significantly increase the VCS efficiency. Figure 1 shows a kinematic scheme of the VCS design with a LIM. Figure $2 \mathrm{~A}$ and B show a general view of the experimental setup and a general view of the working body with sieves.

A working body 3 is placed inside housing 1 . The working body consists of three levels of cylindrical sieves, which receive rotational and vertical oscillatory movements from the electric drives (Linenko et al., 2018).

The electric drive of the working body vibration movement is a flat LIM consisting of several inductors 2 , and a ring 7, which is spring-loaded relative to the base using rollers 8 and elastic elements 9 . The working body 3 receives the rotation from the electric motor 12 with the help of V-belts. When the inductors are connected to the mains, the ring begins to move forward along the vertical plane. During the rotational movement, the elastic elements are compressed. When the inductors are disconnected from the mains, the ring returns to its original state due to the energy accumulated in the elastic elements. Depending on the type, grade, and humidity of the grain mixture, the control unit can regulate the vibration parameters of the working body in the vertical plane according to the frequency and amplitude by changing the duration and frequency of the flat LIM inductors when connected to the mains (Linenko et al., 2018).

Creating a vibrating electric drive of the vibro-centrifugal grain separator with the LIM made it possible to go back from the mechanical motion transformer, reduce power consumption and weight-size parameters.

This technique is useful, but its efficiency can be increased by modifying or removing the expensive and complicated control unit, which periodically connects and disconnects the LIM inductors from the mains during operation. This is accompanied by large starting currents in the windings and consequently reduces the vibrating drive reliability.

The increase in the technical and economic indicators of the VCS with a LIM is associated with the use of new techniques that simplify the electric drive of oscillatory motion by self-vibrating sieves (i.e., without a control unit for periodic LIM switching on and off) (Linenko et al., 2019).

For this purpose, symmetrical through-holes should be made in the flat LIM rotor (ring) (Figure 3A and B). Symmetrical throughholes on ring 7, which is also a LIM rotor, make it possible not to disconnect the LIM inductors from the mains during the VCS operation. Therefore, the LIM inductors are always connected to the 
mains. The rotating ring with holes enters and leaves the magnetic field of the LIM inductors, thus generating vibration. Through the spline joint 10 (Figure 1), the rotational motion is imparted from the rotation engine 12 to ring 7 . During rotation, when a part of the ring without holes is located opposite the LIM inductors (Figure
3B), an electromagnetic force occurs and makes the ring perform a translational motion, for example, down in a vertical plane. In this case, the elastic elements 9 located under the ring are compressed. As ring 7 rotates, at a certain point the symmetrical holes 13 of the ring are opposite the LIM inductors (Figure 3B). Simultaneously,
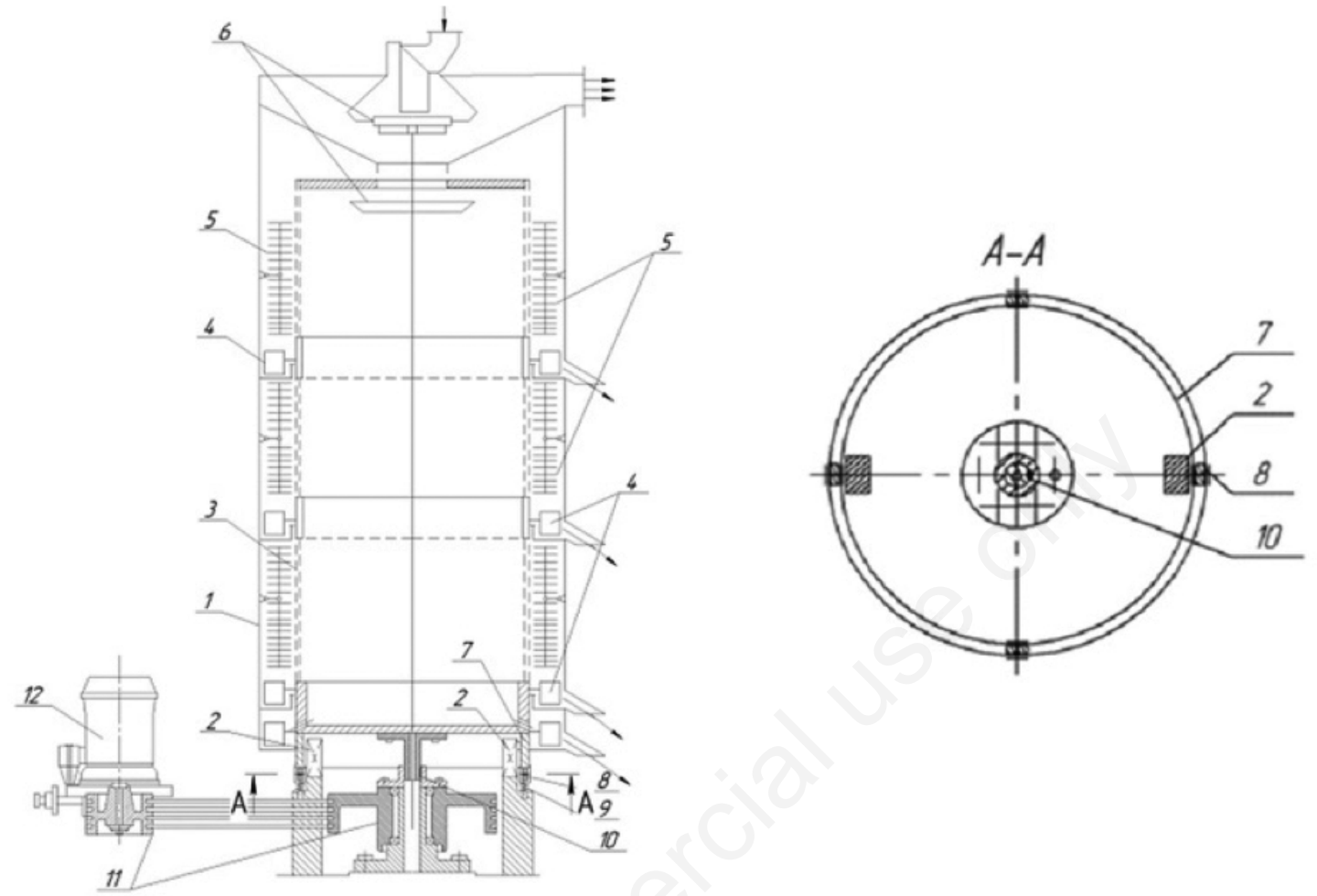

Figure 1. Kinematic scheme of the vibro-centrifugal separators with a linear induction electric motor (LIM) 1-housing, 2-LIM inductors, 3-cylindrical sieves, 4-blades, 5-cylindrical disk cleaners, 6-spreader, 7-ring, 8-rollers, 9-elastic elements, 10-spline connection, 11pulleys, 12-electric motor.
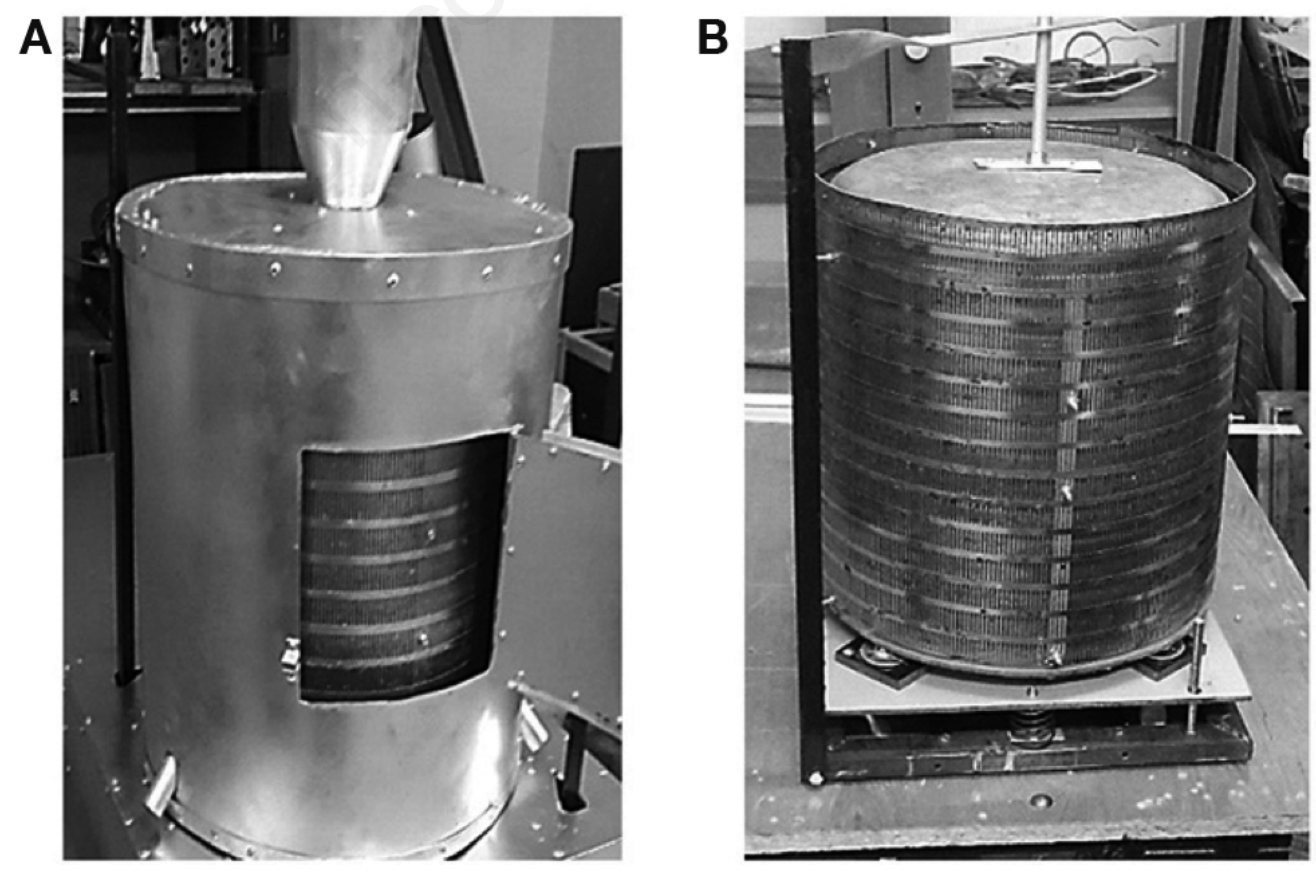

Figure 2. General view of: A) experimental vibro-centrifugal separators installations; B) of the working body with working sieves. 
the electromagnetic field disappears, and, due to the energy accumulated in the elastic elements, the ring returns to its original state and continues to rotate. Then, when parts of the ring without holes pass in front of the inductors, the ring will perform again a translational motion, and the described process will repeat. The oscillation frequency of the working body is determined by the number of holes on the ring and its rotation frequency (Linenko et al., 2019).

Consequently, the proposed technique allows the vibration movement from the LIM of the VCS working body without using a control unit for LIM periodic connection and disconnection from the mains. This significantly reduces the cost and material consumption of the entire construction and increases its reliability.

Another technique makes it possible to change the working body oscillation frequency without changing either the rotation frequency or the rotor design (i.e., the number of symmetrical holes), since the control of the working body oscillation frequency by changing the amount of symmetrical through holes on the rotor (ring) leads to the replacement of the drive functional elements and restricts its use.

If the technology requires to change the vibration parameters of the working body at a constant speed, the following design of the VCS vibrating drive without the block for the LIM inductors switching on and off can be used.

Symmetrical through holes can be made at several levels of the LIM rotor, provided that they are offset from one another. The number of levels of inductors corresponds to the number of levels of through symmetrical holes (Figure 4).

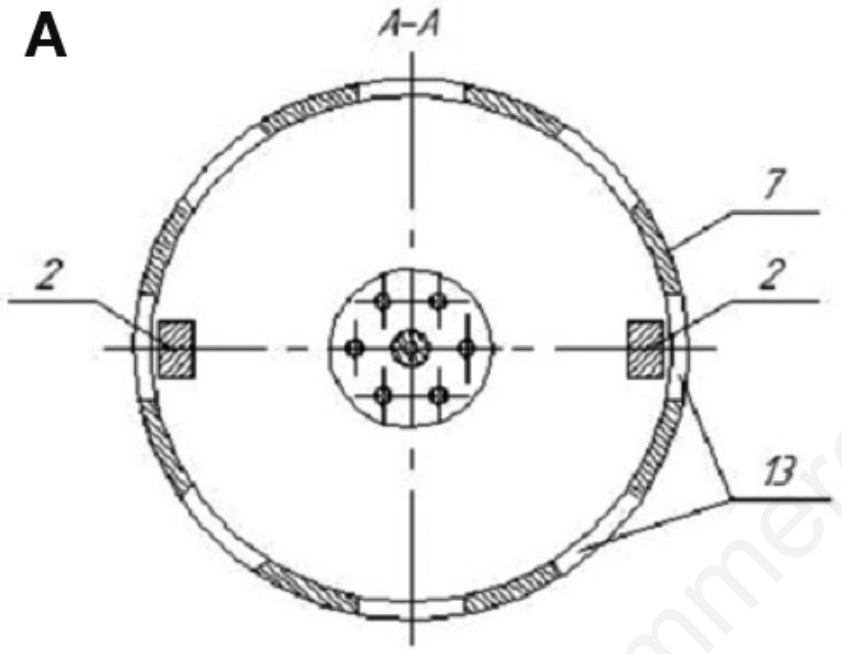

B
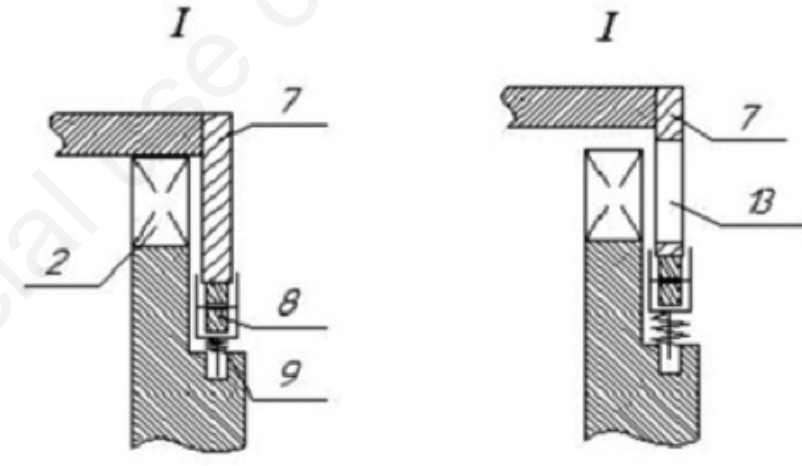

Figure 3. A) General view of the vibrating drive; B) secondary element positions during operation of the vibro-centrifugal separators: 2-linear induction electric motor inductors, 7-ring, 8-rollers, 9-elastic elements, 13-through holes.

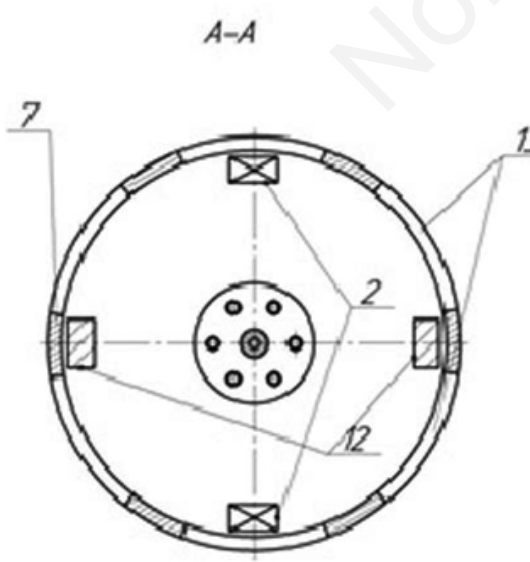

A

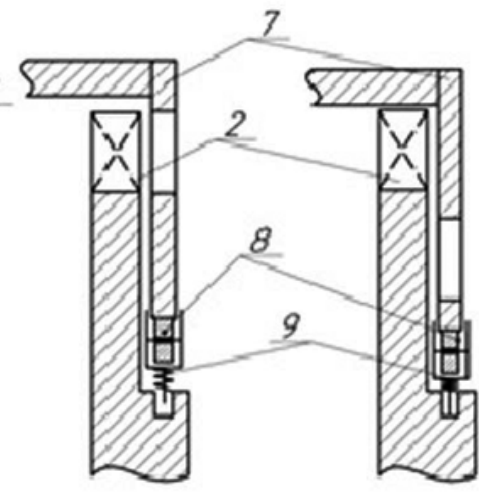

B

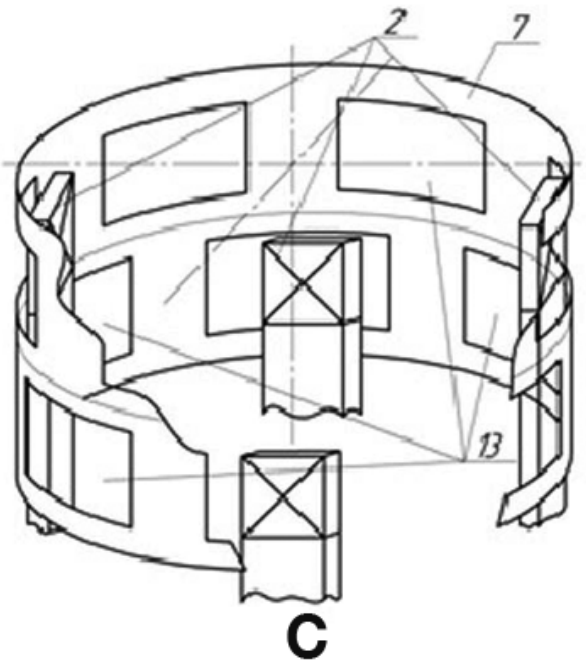

Figure 4. A) General view of the vibrating drive; $B$ and C) secondary element position during the vibro-centrifugal separators operation 2-linear induction electric motor inductors, 7-ring, 8-rollers, 9-elastic elements, 13-through holes. 
During rotation, when a part of the ring without holes is located opposite the LIM inductors of the appropriate level, an electromagnetic force occurs, which makes the ring execute a translational motion, for example, downward along a vertical plane. In this case, the elastic elements located under the ring are compressed. As the ring rotates, there comes the point when the symmetrically located holes of the appropriate ring are opposite the LIM inductors (Figure 3B). Simultaneously, the electromagnetic field disappears, and due to the energy accumulated in the elastic elements, the ring returns to its original state, continuing to make a rotational movement. Then, a part of the next level ring without holes passes by the appropriate level inductors. At the same time, the ring executes a translational motion again, and the described process repeats. The holes on the levels are located at a specific interval. When passing this interval, the ring returns to its original state. The value of the intervals is determined by the required vibration frequency of the working body necessary for the technological process. The working body oscillation frequency is determined by the number of levels of holes on the ring and the number of levels of inductors used for operation, while the rotation frequency of the working body remains constant.

The LIM inductors on the levels are connected in series, but they are energized in parallel. Therefore, during the vibrating drive operation, the number of levels with the inductors connected to the mains can be controlled independently. Moreover, the more levels of inductors connected to the mains, the higher the working body vibration frequency. Consequently, this technique makes it possible to control the separator working body vibration frequency from the LIM, regardless of the working body rotation speed, increasing the installation technological effectiveness.

The proposed approach was studied thoroughly to determine the vibrating drive rational parameters, and an appropriate mathematical model was developed.

The mathematical model was implemented in accordance with the equations of the VCS dynamics, which have the following form:

i) for the translational working body motion:

$m a=F_{L I M}-F_{f r}-F_{r e s}-F_{e l}$

ii) for the working body rotational motion:

$$
J \frac{\partial \omega}{\partial \tau}=M_{A E M}-M_{r e s}-M_{f n^{\prime}}
$$

where $m$ is the mass of the vibrating system (the working body and the LIM secondary element), $\mathrm{kg} ; a$ is the acceleration of the vibrating system, $\mathrm{m} / \mathrm{s}^{2} ; F_{\text {LIM }}$ is the force developed by the LIM, N; $F_{f r}$ is the force of friction in the spline connection (taking into account the mass of the oscillating system, and friction coefficient), N; $F_{e l}$ is the elastic force arising from the deformation of the elastic element (Hooke's law), $\mathrm{N} ; F_{\text {res }}$ is the resistance force of the grain mixture to the sieve movement, $\mathrm{N}$; is the dynamic moment, $\mathrm{N} \cdot \mathrm{m}$; $M_{A E M}$ is the moment developed by the asynchronous electric motor of rotational motion, $\mathrm{N} \cdot \mathrm{m} ; M_{\text {res }}$ is the resistance torque of the grain flow in the working gap, $\mathrm{N} \cdot \mathrm{m} ; M_{f r}$ is the friction torque in the bearings $\mathrm{N} \cdot \mathrm{m}$.

In the mathematical model, the LIM is represented by the ParkGorev equations which are composed on the basis of the substitution scheme (Aipov and Linenko, 2013; Nasar and Boldea, 1981):

$$
\left\{\begin{array}{l}
\frac{\partial \phi_{x 1}}{\partial t}=U_{x 1}-\frac{\pi}{\tau} V_{0} \frac{R_{1} X_{r}}{X_{S} X_{r}-X_{m}} \phi_{x 1}+\frac{\pi}{\tau} V_{0} \frac{R_{1} X_{m}}{X_{S} X_{r}-X_{m}} \phi_{x 2}+\frac{\pi}{\tau} V_{0} \phi_{y 1} \\
\frac{\partial \phi_{y 1}}{\partial t}=U_{y 1}-\frac{\pi}{\tau} V_{0} \frac{R_{1} X_{r}}{X_{S} X_{r}-X_{m}} \phi_{y 1}+\frac{\pi}{\tau} V_{0} \frac{R_{1} X_{m}}{X_{S} X_{r}-X_{m}} \phi_{y 2}+\frac{\pi}{\tau} V_{0} \phi_{x 1} \\
\frac{\partial \phi_{x 2}}{\partial t}=-\frac{\pi}{\tau} V_{0} \frac{R_{2} X_{S}}{X_{S} X_{r}-X_{m}^{2}} \phi_{x 2}+\frac{\pi}{\tau} V_{0} \frac{R_{2} X_{m}}{X_{S} X_{r}-X_{m}^{2}} \phi_{x 1}+\frac{\pi}{\tau}\left(V_{0}-V\right) \phi_{y 1} \\
\frac{\partial \phi_{y 2}}{\partial t}=-\frac{\pi}{\tau} V_{0} \frac{R_{2} X_{S}}{X_{S} X_{r}-X_{m}^{2}} \phi_{y 2}+\frac{\pi}{\tau} V_{0} \frac{R_{2} X_{m}}{X_{S} X_{r}-X_{m}^{2}} \phi_{y 1}+\frac{\pi}{\tau}\left(V_{0}-V\right) \phi_{x 2} \\
F_{L L M}=\frac{3}{2} \frac{\pi V_{0}}{\tau} \frac{X_{m}}{X_{S} X_{r}-X_{m}^{2}}\left(\phi_{x 2} \phi_{y 1}-\phi_{x 1} \phi_{y 2}\right) ; \frac{\partial V}{\partial t}=\frac{\left(F_{L M}-F_{f r}\right)}{m}
\end{array}\right.
$$

where $U_{X 1}, U_{Y 1}$ is the voltage inductor along the $O X, O Y, V$ axes; $\tau$ - LIM is the winding pole division; $X_{S}=X_{1}+X_{m}, X_{r}=X_{2}+X_{m}$ are the values of resistance, the inductor and the secondary element used in the model, Ohm; $R_{1}, X_{1}, R_{2}, X_{2}$ are the active and reactive resistance of inductor and the secondary element in the winding of inductor respectively, Ohm; $X_{m}$ is the resistance of the mutual induction between the inductor and the secondary element, Ohm, $V_{o}$ LIM is synchronous speed, $\mathrm{m} / \mathrm{s} ; \varphi_{X 1}, \varphi_{Y 1}, \varphi_{X 2}, \varphi_{Y 2}$ is the flux linkage along the axes $O X, O Y$ of the inductor and secondary element, respectively, $\mathrm{Wb} ; V$ is the speed of the LIM secondary element, $\mathrm{m} / \mathrm{s}$.

In grain separation, the quality of grain mixture separation is very important. Therefore, it is necessary to justify the electric drive parameters and determine the installation capacity (Boac et al., 2014; Kharchenko, 2015). Figure 5 shows the grain mixture distribution on the sieve.

The grain mixture is fed by the spreader to the inner surface of

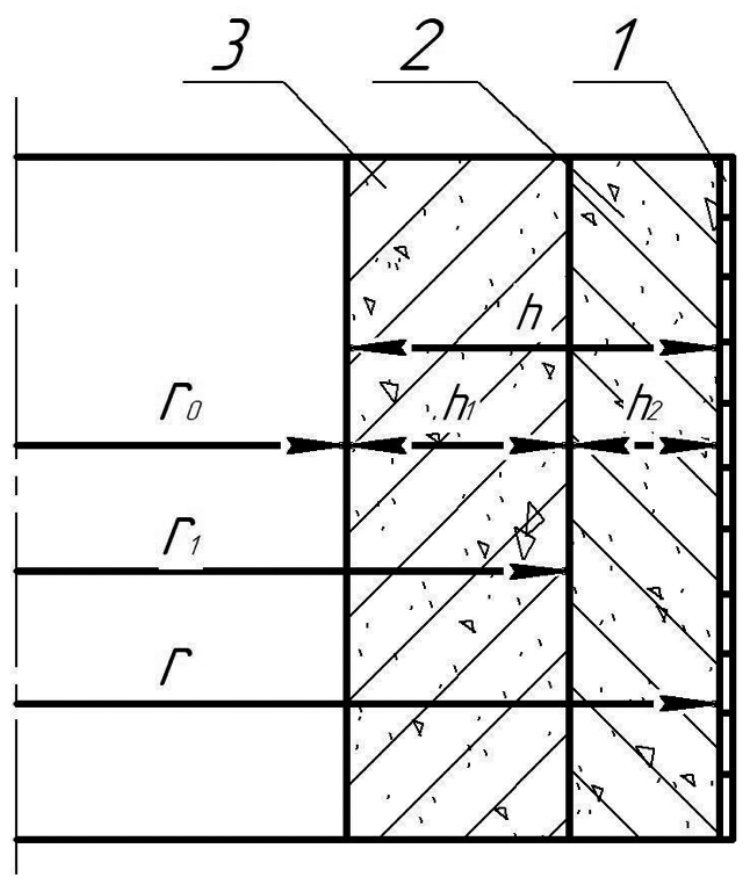

Figure 5. Grain mixture distribution over the cylindric sieve surface 1-sieve, 2-, 3-layers of coarse and fine grain mixture, respectively. 
the receiving section of the upper part of the sieve, forming a cyclic layer. As a result of gravity and vibrations, the mixture moves down. The free surface of the mixture layer forms a cylindrical surface with radius $r_{0}$. A layer of larger grain particles located closer to the sieve surface forms a cylindrical surface with radius $r_{l}$ (Tishchenko et al., 2010).

A simplified model version, in which the cyclic layer of variable viscosity is divided into two homogeneous cyclic sublayers (Figure 5) with different dynamic viscosity coefficients $\mu$ (Linenko et al., 2018; Tishchenko et al., 2010), was used to determine the productivity $Q$ of the VCS (4) for food grains.

A viscosity coefficient $\mu_{1}$ of the sublayer closer to the sieve surface is higher than the viscosity coefficient $\mu_{2}$ of the sublayer that borders on the grain-free surface.

$$
Q=\pi\left\{\begin{array}{l}
\left(r_{1}^{2}-r_{0}^{2}\right) \cdot\left[\begin{array}{l}
\frac{\rho g}{\mu_{1}} \\
\frac{1}{2}\left(r_{1}^{2}-r_{0}^{2}\right)-\frac{\rho g}{\mu_{1}} \cdot r_{0}^{2}+\left(\begin{array}{l}
\frac{\rho g}{\mu_{2}} \cdot r^{2}-\frac{\rho g}{2 r} \cdot\left(r_{1}^{2}-r_{0}^{2}\right)+ \\
\frac{\rho g}{\mu_{2}}-\frac{\rho g}{\mu_{1}} \\
\left.+\frac{\rho g}{2} \cdot\left(\frac{r_{1}^{2}}{2}-r_{0}^{2} \cdot \ln \frac{r_{1}}{r}\right)\right)
\end{array}\right]+r_{0}^{2} \cdot\left(r_{1}^{2} \cdot \ln \frac{r_{1}}{r}-r_{0}^{2} \cdot \ln \frac{r_{0}}{r}\right)+\left(r_{1}^{2}-r^{2}\right) . \\
\frac{\frac{\rho g}{2}}{\frac{\mu_{2}}{8}} \cdot\left(r^{2}-r_{0}^{2}\right)+\frac{\rho g}{\frac{\mu_{2}}{4}} \cdot r^{2}-\frac{\rho g}{2 r} \cdot\left(r^{2}-r_{0}^{2}\right)-\frac{\rho g}{\frac{\mu_{2}}{2}} \cdot r_{0}^{2}
\end{array}\right]-\frac{\rho g}{\frac{\mu_{2}}{2} \cdot r_{0}^{2} \cdot r_{1}^{2} \cdot \ln \frac{r_{1}}{r}}
\end{array}\right\},
$$

The dynamic viscosity coefficient $\mu$ of the grain mixture (5) is determined by the working body amplitude and vibration frequency, which are set by the rigidity of the vibrating drive elastic elements:

$$
\mu=\frac{4 f p r_{c}^{2}}{v \cdot\left(\left(-\frac{c}{m \cdot a}\right)^{2}-\left(\frac{3}{\pi}\right)^{2} \cdot\left(\frac{p}{v^{2} \cdot r_{c} \cdot \rho}\right)^{2} \cdot f_{c}^{2}\right)^{1 / 2}},
$$

where $p$ is the pressure of the grain mixture, $\mathrm{Pa} ; f$ is the coefficient of the grain mixture friction on the sieve surface; $\rho$ is the grain mixture layer density $\mathrm{kg} / \mathrm{m}^{3} ; c$ is the elastic element rigidity, $\mathrm{N} / \mathrm{m}$; $m$ is the oscillatory system mass, $\mathrm{kg} ; a$ is the working body acceleration, $\mathrm{m} / \mathrm{s}^{2}, v$ is the oscillation frequency of the working body, $\mathrm{Hz} ; r_{c}$ is the radius of a single grain particle, $\mathrm{m} ; f_{c}$ is the coefficient of dry friction between grain particles.

The mathematical model of the developed VCS vibrating drive is implemented using the MatLab (Simulink) object-visual modelling environment (Figure 6).

Since the contribution of small and medium-sized farms to the national food security is increasing every year, the development of lowproductivity technological machines is relevant. In this regard, the parameters of the LIM replacement scheme for an installation with a capacity of up to 6 tons of grain chaffer were determined to study the mathematical model: $R_{l}=4.08 \mathrm{Ohms}, X_{l}=0.145 \mathrm{Ohms}, X_{2}=0.9 \mathrm{Ohms}$, $X_{m}^{\prime}=4.7 \mathrm{Ohms}, R_{2}=5.0 \mathrm{Ohms}$, and pole division $=0.036 \mathrm{~m}$.

The following values were taken as basic values for the study: the grain mixture density is $730 \mathrm{~kg} / \mathrm{m}^{3}$ (parameters of wheat grain with a humidity of $16 \%$ ); the nominal working body angular speed

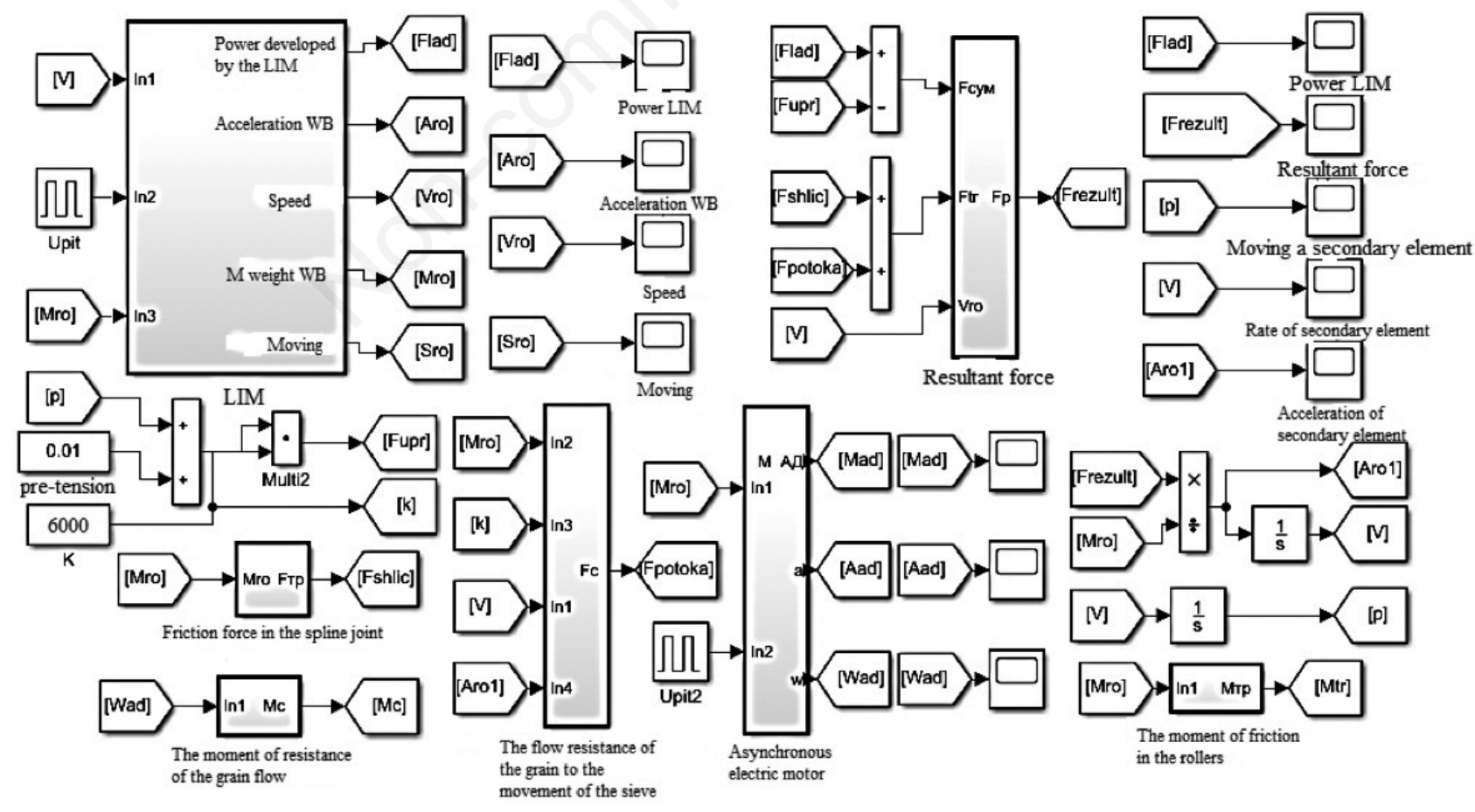

Figure 6. Visualization of the main window of the mathematical model of the vibro-centrifugal separators (VCS) with a linear induction electric motor (LIM) in Matlab (Simulink). 
is $\omega=11.5 \mathrm{rad} / \mathrm{s}(110 \mathrm{rpm})$; the working body mass $m_{l}$ is $15 \mathrm{~kg}$; the grain mixture mass $m_{2}$ is $1.5 \ldots 8.0 \mathrm{~kg}$. Based on productive capacity, the rigidity of the elastic elements $c$ is $5000 \mathrm{~N} / \mathrm{m}$, which provides the required vibration parameters of the working body.

\section{Results and discussion}

The optimal seed separation kinematic mode for a particular grain crop has its own specific combination of values of the working body vibration frequency and amplitude that agree quite well with each other (Zorawski et al., 2014; Ma et al., 2016). Determining the amplitude-frequency characteristics depending on the drive design parameters is an important research task, since the obtained dependencies can be used to design specific structures.

For high-quality seed separation when changing the type of agricultural crop, it is necessary to adjust the oscillation amplitude $A_{\text {osc }}$, and the VCS working body oscillation frequency $v$. An effective way to regulate these parameters in the proposed technical solutions is to change the rigidity of the elastic element (the most labour-intensive), the working body rotation speed $\omega$, and the number of holes in the ring.

The working body oscillation frequency $v$ and the oscillation amplitude $A_{\text {osc }}$ are determined by the working body rotation frequency $\omega$ and the number of symmetrical holes in the ring, taking into account the rigidity of the elastic elements $c$ and the oscillating system mass $M=m_{1}+m_{2}$.

Figure 7 shows the dependence of the working body vibration amplitude and frequency on its rotation frequency which in turn depends on a different number of through symmetrical holes in the secondary element.

As can be seen from this graph, the working body vibration frequency has a linear dependence on its rotation frequency. Simultaneously, the higher the number of symmetrical holes in the secondary element, the higher the working body vibration amplitude. A secondary element with 4 through holes is the most preferred option. This solution ensures the working body vibration frequency to be $10-12 \mathrm{~Hz}$, when the nominal angular speed of the working body $\omega$ is $11.5-12 \mathrm{rad} / \mathrm{s}(110-120 \mathrm{rpm})$.

The working body oscillation parameters, in turn, depend on the oscillating system mass. Figure 8 shows the graphs of the VCS amplitude-frequency characteristics, when the grain mixture mass on sieves changes. The working body mass is $m_{l}=15 \mathrm{~kg}$, the grain mixture mass $m_{2}$ ranges from 4 to $6 \mathrm{~kg}$.

As Figure 8 shows, a 10\% increase or decrease in the oscillating system mass does not significantly affect the VCS amplitudefrequency parameter. Therefore, a change in the load during the drive operation does not violate the law of the working body vibrations and ensures the quality of the technological process.

A pre-set VCS productive capacity is provided by the corre-

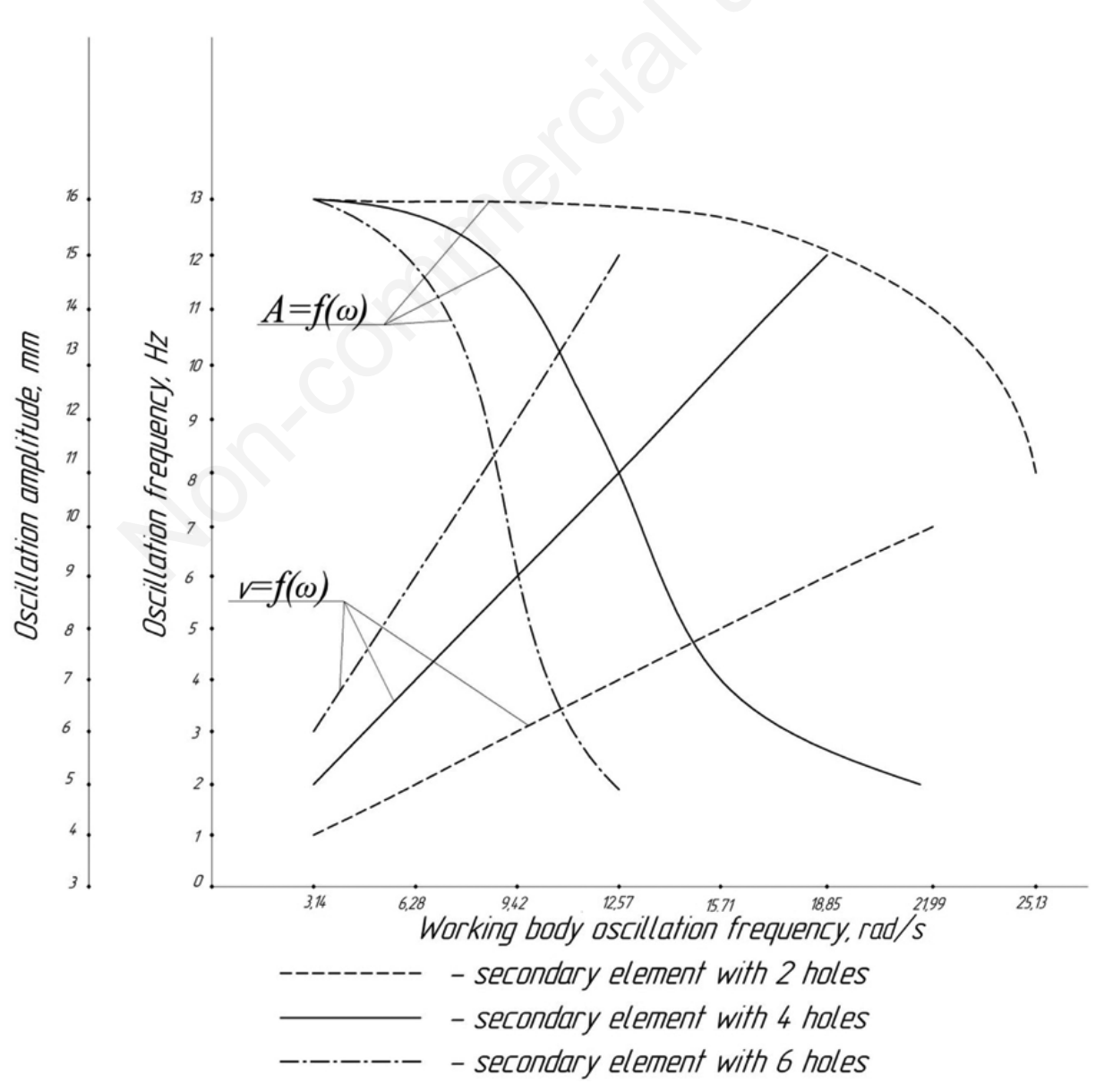

Figure 7. Dependences of the working body vibration amplitude and frequency on the frequency of its rotation which in turn depends on a different number of through symmetrical holes in the secondary element. 
sponding force developed by the LIM. Figure 9 shows the dependence of the force developed by the LIM on the VCS productive capacity with different grain layer thicknesses on the sieves. Simplified grain mixture movement models were used for mathematical modelling. So, for example, a single-layer model was considered for seed grains, a two-layer model for food grains, and a three-layer model for grain chaffer. These types of grains differ in their composition in terms of degree of contamination and linear size of the particles that make up the mixture. The freshly harvested grain mass, called grain heap, is has a diverse composition and a large number of weed impurities that differ from the grains of the main crop in terms of linear dimensions several times, in larger or smaller directions. Food grains, which are characterized by high quality and the absence of weed impurities, can include a certain quantity of broken particles, unlike seed grains. These parameters determine the thickness of the grain layer on the sieve and the amount of energy spent for processing the grain mixture. This dependence makes it possible to choose the most rational LIM parameters at the installation design stage, which can ensure the efficiency of the technological process of the grain mixture separation with minimal energy costs.

Consequently, the analysis of the resulting mathematical model (3) makes it possible to choose the most rational parameters for the rigidity of the elastic elements, the LIM power, and the operation mode, when designing the VCS.

According to the kinematic scheme shown in Figure 1, and

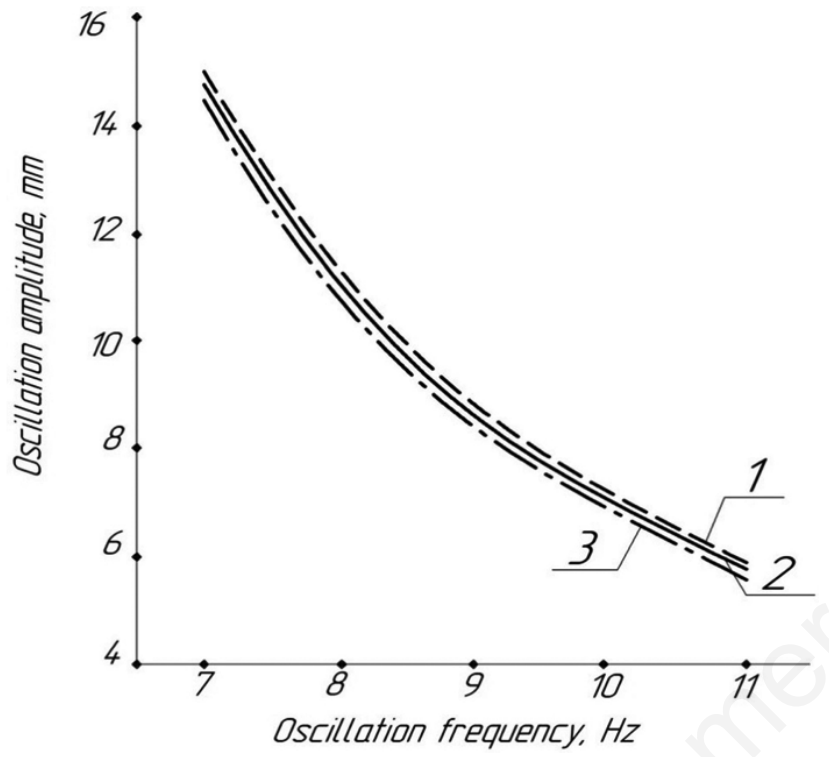

Figure 8. Vibro-centrifugal separators (VCS) amplitude-frequency characteristics depending on the oscillating system mass, when the number of holes in the secondary element is equal to 4: 1$M=19 \mathrm{~kg}, 2-\mathrm{M}=20 \mathrm{~kg}, 3-\mathrm{M}=21 \mathrm{~kg}$.

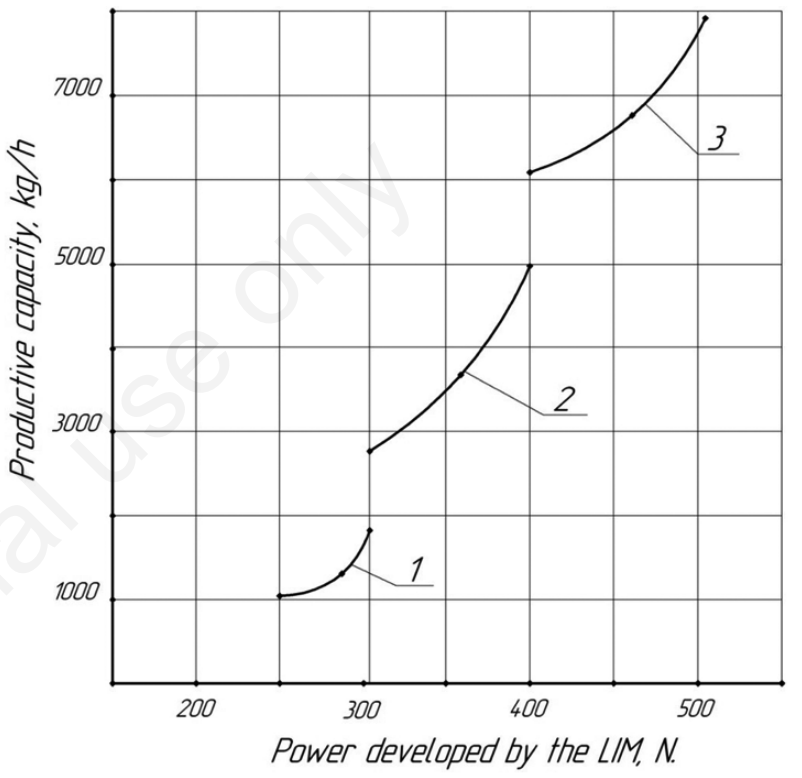

Figure 9. Dependence of the vibro-centrifugal separators (VCS) productive capacity on the developed linear induction electric motor (LIM) power: 1-seed grain, 2-food grain, 3-coarse separation (grain chaffer).
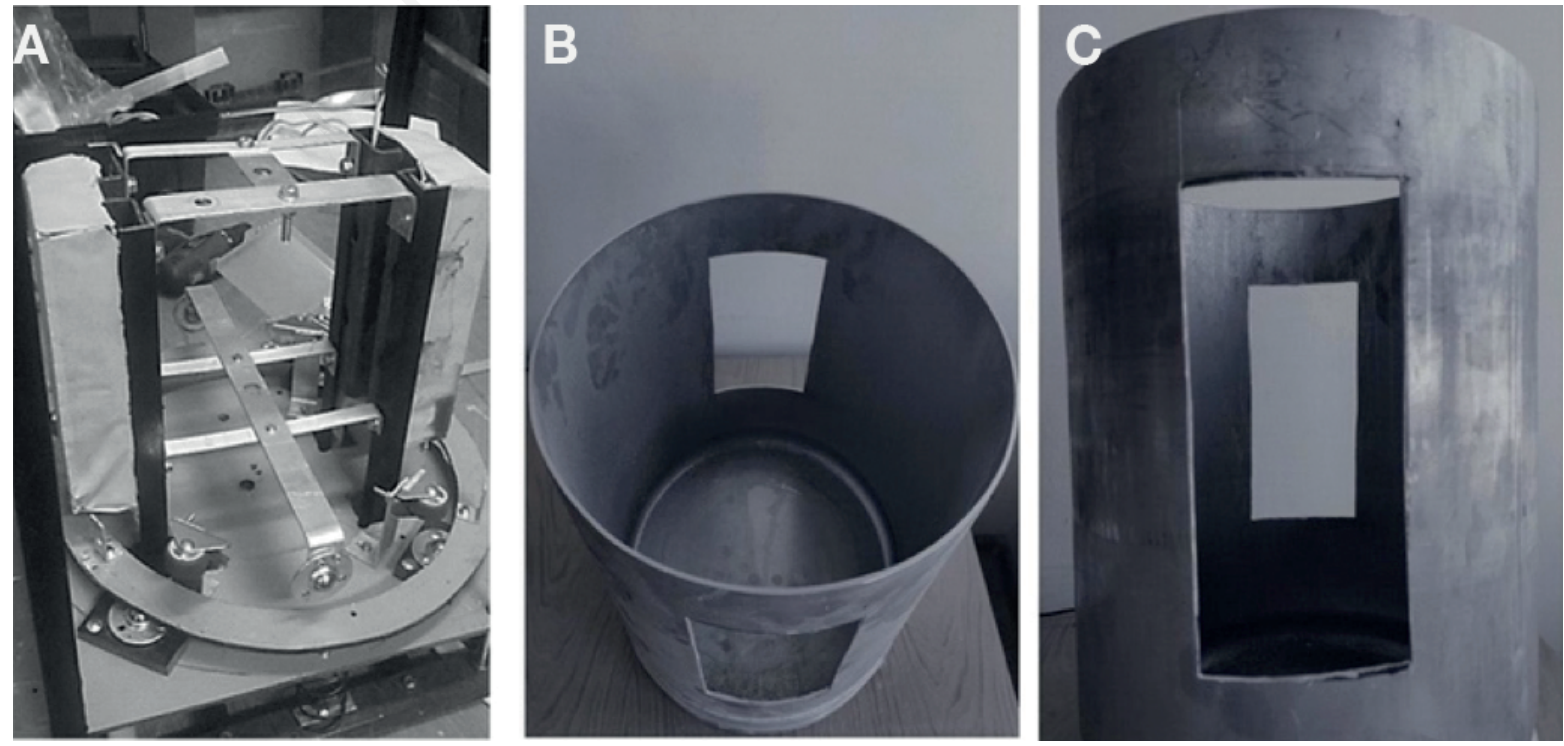

Figure 10. General view of: A) is the flat linear induction electric motor inductors, the secondary element with 2 (B) (C) through holes. 
based on a flat LIM, a working VCS experimental installation with a vibrating drive was designed, in which case, the secondary element has 2 or 4 through symmetrical holes (10). Experimental studies were conducted to verify the adequacy of the developed mathematical mode according to the vibrating drive amplitude-frequency parameter.

The difference between the obtained experimental and calculated dependencies did not exceed 6\% (Figure 11).

Therefore, the developed mathematical model proved to be adequate and can be used in practice.

\section{Conclusions}

New original designs of the VCS vibrating drives were proposed. They can provide adjustable working body vibrations within the required range without using the control assembly of periodic LIM switching on and off from the mains. A mathematical LIM model was developed and studied to determine the rational parameters of the VCS operation. The LIM secondary element with 4 through holes provided the required oscillation frequency of 10-12 $\mathrm{Hz}$ at a working body rotation speed of $11 \mathrm{rad} / \mathrm{s}$. A formula for determining the VCS productive capacity was proposed considering the presence of elastic elements in the vibrating drive. The LIM was implemented using the Park-Gorev differential equations. The driving characteristics of a VCS with a linear electric drive were analyzed using a mathematical model in the Matlab object-visual modelling environment (Simulink).

It was calculated that a $10 \%$ increase or decrease in the oscillating system mass did not significantly affect the VCS amplitudefrequency parameter. Therefore, a change in the load during the drive operation in a force-oscillation regime did not violate the law of the working body vibrations and ensured the quality of the technological process.

A comparison of theoretical and experimental dependencies showed that their maximum discrepancy did not exceed $6 \%$, which proved that the resulting mathematical model adequately reflected the physical processes, and could be used in practical calculations. The results of the study do not contradict the provisions presented in the publications on the development of grain separators (Tishchenko et al., 2010; Ospanov et al., 2019).

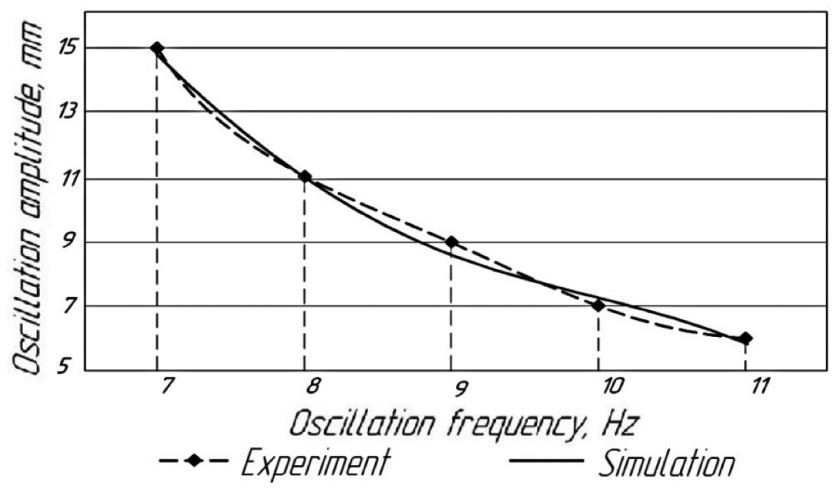

Figure 11. Comparison of the simulated amplitude-frequency characteristic with the experimental characteristic at the mass of the oscillatory system $M=20 \mathrm{~kg}$.
The proposed technical solution creates a vibrational motion of the centrifuge working body from the LIM without using a control unit for its periodic connection and disconnection from the mains. This can reduce the starting currents in the inductor windings and increase the reliability of the installation. Thanks to the use of the LIM in the VCS vibrating drive, it is possible to save metal. The LIM's power for a production capacity of $6000 \mathrm{~kg} / \mathrm{h}$ is $2.8 \mathrm{~kW}$, which is $28 \%$ less compared to the existing VCS installation with the same production capacity. The service intervals of the vibrating drive are increased from 180 to 250 hours, which accounts for $37.8 \%$. As a result, the goal of the research was achieved.

\section{References}

Ahmadinia N. 2014. The linear induction motor (LIM) \& single linear induction motor (SLIM). Int. J. Electr. Power Energy Syst 3:71-5.

Aipov R.S., Linenko A.V. 2013. Linear electric machines and linear asynchronous electric drives of technological machines. Textbook. Bashkir State Agrarian University, Ufa, Russian Federation.

Alanis A.Y., Rios J.D., Rivera J., Arana-Daniel N., Lopez-Franco C. 2015. Real-time discrete neural control applied to a Linear Induction Motor. Neurocomputing 164:240-51.

Al Maidi A.A. 2015. Ways to increase and improve the efficiency of grain production. Young Sci. 4:296-9.

Boac J.M., Ambrose R.P.K., Casada M.E., Maghirang R.G., Maier D.E. 2014. Applications of discrete element method in modeling of grain postharvest operations. Food Eng. Rev. 6:128-49.

Ganesh Sampath V., Abhishek K., Lenin N.C. 2016. Design, development and electromagnetic analysis of a linear induction motor. Appl. Mech. Mater. 852:794-8.

Iarullin R.B. 2007. Dynamics of vibrating grain separators with adjustable parameters. Problems of electric drive. Ufa State Academy of Economics and Service, Ufa, Russian Federation.

Jiao Z.X., He P., Yan L., Liang H.S., Wang T.Y. 2017. Hybrid thermal modeling of tubular linear oscillating motor based on sectionalized equivalent thermal circuit. Int. J. Appl. Electrom. Mech. 54:535-51.

Kharchenko S. 2015. Modeling the dynamics of the grain mixtures with the screening on cylindrical vibrating sieve separators. TEKA. Comm. Motorizat. Energ. Agricult. 15:87-93.

Khasanov E.R., Gabitov I.I., Mudarisov S.G., Khamaletdinov R.R., Rakhimov Z.S., Akhmetyanov I.R., Farkhutdinov I.M., Masalimov I.H., Musin R.Z. 2019. Justification of parameters of seed treater with an eccentrically fixed drum influencing the motion character and seed treatment modes. Bulg. J. Agric. Sci. 25:119-28.

Lim J., Jeong J.-H., Kim C.-H., Ha C.-W., Park D.-Y. 2017. Analysis and experimental evaluation of normal force of linear induction motor for maglev vehicle. IEEE T. Magn. 53:8300504.

Linenko A.V., Aipov R.S., Yarullin R.B., Gabitov I.I., Tuktarov M.F., Mudarisov S.G., Kabashov V.Y., Kamalov T.I., Gilvanov V.F., Khalilov B.R. 2018. Experimental vibro-centrifugal grain separator with linear asynchronous electric drive. J. Appl. Eng. Sci. 13:6551-7.

Linenko A.V., Khalilov B.R., Kamalov T.I., Khusnutdinov S.I. 2019. Vibro-centrifugal separator. Patent 2686760. 30.04.2019. Application No. 2018125563 DTD 11.07.2018, Bashkir State Agrarian University, Ufa, Russian Federation. 
Liu X.-P., Zhang Y.-L., Yang D. 2014. Finite element analysis of 5XF150/180 type grain cleaning machine. In: 2014 International Conference on Mechanism Science and Control Engineering (MSCE). DEStech Publications, Inc., Lancaster, Pennsylvania, USA, p 112.

Ma X.-D., Zhang Y.-B., Liu Y., Zheng X.-W. 2016. Simulation of grain segregation under horizontal rotational oscillations. Granul. Matter 18:1-6.

Nasar S.A., Boldea I. 1981. Linear motion electric machines. Wiley, New York, NY, USA.

Ospanov A., Muslimov N., Timurbekova A., Jumabekova G., Almaganbetova A., Zhalelov D., Nurdan D. 2019. The study of indicators of the quality test of poly-cereal whole meal flour for making pasta. J. Hyg. Eng. Des. 27:32-8.

Ostrikov A.N., Ospanov A.A., Shevtsov A.A., Muslimov N.Z., Timurbekova A.K., Jumabekova G.B. 2020. Mathematical model of high-temperature tube-shaped pasta drying in a conveyer belt drier. Int. J. Food Eng. [Epub ahead-of-print].

Ostrikov A., Ospanov A., Vasilenko V., Muslimov N., Timurbekova A., Jumabekova G. 2019. Melt flow of biopolymer through the cavities of an extruder die: Mathematical modelling. Math. Biosci. Eng. 16:2875-905.

Panasiewicz M., Sobczak P., Mazur J., Zawiślak K., Andrejko D. 2012. The technique and analysis of the process of separation and cleaning grain materials. J. Food Eng. 109:603-8.

Pathan A.S., Waghmare P.B., Shaikh A.M., Salunkhe P.V., Raut M., Jadhav V.K. 2017. Design of linear induction motor works as conveyer. Int. Eng. Res. J. Special Issue:279-83.

Sannomiya K., Morizane T., Kimura N., Omori H. 2017. Experimental confirmation of thrust and attractive force control for linear induction motor by two different frequency components. Proc. 2017 11th Int. Symp. Linear Drives Ind. App. (LDIA), IEEE, Osaka, Japan.

Tishchenko L.N., Mazorenko D.I., Piven M.V., Kharchenko S.A., Bredikhin V.V., Mandryka A.V. 2010. Modeling of grain separator processes. Monograph. Kharkov National Technological University of Agriculture, "Miskdruk", Kharkov, Ukraine.

Vinod K. 2017. Design of linear induction motor works as conveyer. Int. Eng. Res. J. 1:279-83.

Zorawski D., Dzikowska W., Peszynski K. 2014. Modelling of the vibrating dryer drive system. Proc. 20th Int. Conf. Eng. Mech. (EM), Svratka, Czech Republic. 